\title{
In memoriam Liselotte Neis
}

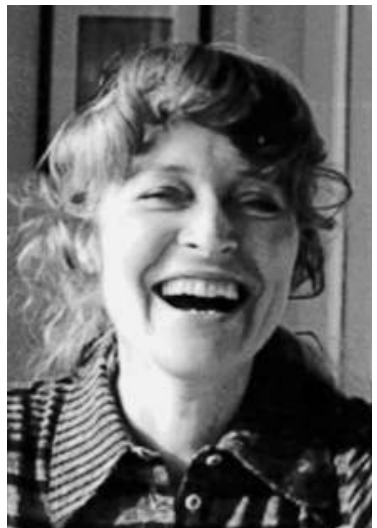

Dr. med. Liselotte Neis $(†$ 2013)
Die Pionierin der Verhaltenstherapie (VT) in der Romandie, Dr. med. Liselotte Neis, ist im Februar 2013 in vorgerücktem Alter verstorben.

Die vielseitige und ihren Verpflichtungen stets ergebene Psychiaterin und Psychotherapeutin hatte 1952 ihr Medizinstudium mit dem Staatsexamen und der Promotion an der Christian Albrechts-Universität Kiel abgeschlossen. Ihre Erfahrungen in verschiedenen Bereichen der Medizin gewann sie in Hamburg (Innere Medizin), Agra/Lugano (Lungenheilstätte), Shushtar (Iran, Allgemeines Krankenhaus) und Heidelberg (Dermatologie). Zurück in Hamburg, studierte sie von 1959-1962 an der Universität bei Prof. Peter Hofstätter Psychologie, der im deutschen Sprachraum die empirisch fundierte Sozialpsychologie bahnbrechend anregte. Gleichzeitig bildete sie sich in der Psychiatrie und Psychotherapie weiter, u.a. mit einer Lehranalyse, und vertiefte ihre Kenntnisse an der Kantonalen Psychiatrischen Klinik St. Pirmisberg (SG). Seit 1964 hat sie in der Clinique psychiatrique universitaire (Hôpital de Cery) am Département Universitaire de Psychiatrie Adulte der Universität Lausanne gewirkt.

Ende der 60er Jahre begann sich Lilo Neis in die Verhaltenstherapie einzuarbeiten und einschlägige Kurse im Ausland bei namhaften Vertretern dieser damals auch in Europa aufkommenden Therapierichtung zu besuchen. Die neuerworbenen Kenntnisse und Kompetenzen vermittelte sie, zusammen mit anderen Expertinnen und Experten, als eine der ersten Verhaltenstherapeutinnen im französischen Sprachraum in Kompaktkursen an junge Psychiater und Psychologen, gekoppelt mit wöchentlichen Fallseminarien im Rahmen der Psychiatrischen Universitäts-Poliklinik von Lausanne. Sie kooperierte auch mit dem Psychologischen Institut der Universität Lausanne, wo sie die Einführung in die VT, Supervision von Fällen und Selbstkontrollgruppen mit Studierenden betreute. In diesem Ansatz theoretisch und praktisch geschult, arbeitete sie an der Weiterbildung und Supervision von Psychiatern und von Psychologen mit, von denen viele später den Fachtitel für Psychotherapie von der Föderation der Schweizer Psychologinnen und Psychologen (FSP) erhielten.

Mit dem Psychologischen Institut der Universität Fribourg verband sie seit 1982 bis Herbst 1995 eine engagierte Zusammenarbeit. Sie bot Pflichtkurse zur Verhaltenstherapie, sowohl methoden- wie auch störungsorientiert, im Rahmen des Curriculums «Überblick über psychotherapeutische Interventionsmethoden» an.

Lilo Neis war nicht nur eine begabte Lehrerin, sie war auch ein Vorbild als gute Therapeutin. Sie wusste die Patienten zu motivieren, setzte Ansprüche an ihre aktive Mitarbeit, Ansprüche, die sie auch an sich selber stellte: Sie bereitete jede Therapiestunde sorgfältig vor und nach, bezüglich der therapeutischen Ziele, möglicher Schwierigkeiten im Prozessgeschehen und allfälliger Adaptationen der therapeutischen Methode.

Ihre wissenschaftlichen Interessen vermochte sie durch ihre Kooperation in mehreren Forschungsgruppen zu verfolgen. In diesen Kontexten war sie ergänzend zu eigenen Veröffentlichungen - Koautorin verschiedener beachtenswerter Publikationen. Dazu gehören z.B. Neis, Imobersteg (1981): Phobie des soins dentaires. Exemple d'une collaboration thérapeutique entre dentiste et psychiatre.

Sie hat mehrere wissenschaftliche Artikel zur Schizophrenie geschrieben. Lilo Neis trug wesentlich zur französischen Anpassung des ersten integrativen Programms der Psychologischen Therapie für Schizophrenie bei, das in der Forschergruppe um Hans Dieter Brenner an der Universitäts- und Poliklinik für Psychiatrie Bern entwickelt worden ist: Pomini, Neis, Brenner, Hodel, Roder (1998): Thérapie psychologique des schizophrénies. Ihr letzter Beitrag datiert von 2002, zusammen mit Pomini und Perrez hatte sie am Kapitel über die Synthese von Verhaltens- und kognitiver Therapie für die von Nicolas Duruz herausgegebene Publikation «Traité de psychothérapie comparée» mitgewirkt.

Wie auch ihre Schwester, die als bildende Künstlerin an der Kunstakademie in Ann Arbor unterrichtete, war die belesene und sprachlich begabte Verstorbene selbst schöpferisch tätig. Sie verfasste Märchen und übersetzte Kinderbücher aus dem Deutschen ins Französische, zusammen mit Anne Salem-Marin für den Verlag La Joie de Lire (u. a. Goethe, Erlbruch: Das Hexen-Einmal-Eins/Cuisine de Sorcière; Hohler, Berner: Wenn ich mir etwas wünschen könnte/Les trois vœux de Barbara).

Wer das Privileg ihrer Freundschaft geniessen durfte, wurde mit spannenden, schönen Briefen und Geschichten beschenkt; auch konnte er sich an ihrer kritischen und gleichzeitig humorvollen Weltbetrachtung, die ihre eigene professionelle Aktivität einschloss, an einem liebevollen Austausch und einer unerschöpflichen Quelle literarischer kreativer Inspirationen erfreuen. Kein Wunder, dass ihre beiden Töchter in ihren Berufen das Reich der Musik und der Bildenden Kunst aufgesucht haben! 